\title{
Comparación del sistema ProTaper Universal con homólogo adulterado: estudio descriptivo
}

\author{
Comparison of ProTaper Universal system with adulterated homologous: descriptive study
}

Gabriela Castro-Núñez ${ }^{1 a b}$, Wilfredo Escalante-Otárola ${ }^{1 a}$, Kennia Scapin Viola ${ }^{1 a b}$, Hernán Coaguila-Llerena ${ }^{2 b}$, Gisele Faria ${ }^{1 c}$, Milton Carlos Kuga ${ }^{1 c}$

\section{RESUMEN}

Objetivos: Describir las características macroscópicas, microscópicas y la caracterización del material de instrumentos rotatorios de Níquel-Titanio (NiTi), ProTaper Universal, en comparación con instrumentos similares de dudosa procedencia. Material y métodos: El análisis macroscópico se realizó por medio de fotografía macro de las cajas e instrumentos. Para el análisis microscópico, se utilizaron los instrumentos S1 y F1, los cuales fueron seccionados y analizados a través de Microscopía electrónica de barrido comparando las partes activas y las secciones transversales con una magnificación de 300 a 550X. Adicionalmente, se realizó la caracterización de los fragmentos, a través de microanálisis por dispersión de energías de rayos-X (EDX). Resultados: $\mathrm{Al}$ análisis macroscópico, las cajas de los sistemas presentaron diferencias en el diseño y especificaciones, los vástagos de los instrumentos presentaron diferencias de forma, color y acabado, y las partes activas de algunos instrumentos, presentaron diferencias de diámetro, forma y acabado. Al análisis microscópico, los instrumentos adulterados presentaron ángulos de corte menos afilados y ausencia de convexidades triangulares. Al comparar la composición química de los instrumentos, el análisis $E D X$ mostró que ambos instrumentos tienen una composición Ni-Ti similar. Conclusiones: Las mayores diferencias entre el sistema ProTaper Universal y su homólogo adulterado se presentaron en el análisis macroscópico. El análisis microscópico presentó diferencias principalmente en el formato de la sección transversal.

PALABRAS CLAVE: endodoncia, instrumentos dentales, preparación del conducto radicular, fotografía, microscopía electrónica.

\section{SUMMARY}

Objectives: To describe the macroscopic, microscopic characteristics and material characterization of nickel titanium (NiTi) ProTaper Universal system scompared to similar instruments of dubious origin. Material and methods: Macroscopic analysis was performed using macro photography of the boxes and files. For microscopic analysis, S1 and F1 files were used, which were sectioned and analyzed by scanning electron microscopy comparing

\footnotetext{
${ }^{1}$ Facultad de Odontología de Araraquara, Universidad Estatal Paulista, São Paulo, Brasil.

2 Facultad de Estomatología, Universidad Peruana Cayetano Heredia. Lima, Perú.

a Magister en Odontología

${ }^{\mathrm{b}}$ Especialista en Endodoncia

c Profesor adjunto
} 
active parts and cross-sections at 300-550X magnification. Additionally, the characterization of fragments was performed by energy dispersive x-ray microanalysis (EDX). Results: Macroscopic analysis showed that boxes differed in design and specifications. File shanks showed differences in shape, color, finish. The active part of some files showed differences in diameter, shape and finish. Microscopic analysis indicated that adulterated files showed less sharp cutting angles and absence of triangular convexities. By EDX, comparing the chemical composition of the instruments, it was shown that both instruments have a composition Ni-Ti like. Conclusions: The major differences between ProTaper Universal system and its counterpart adulterated, were presented in macroscopic analysis. Microscopic analysis showed differences mainly in cross-section configuration.

\section{KEYWORDS: endodontics, Ni-Ti, photography, microscopy, x-ray microanalysis.}

\section{INTRODUCCIÓN}

Los instrumentos endodónticos fabricados con aleaciones de Niquel-Titánio (NiTi) fueron introducidos en el mercado. En 1988 con el objetivo de sustituir la rigidez, es decir, el alto módulo de elasticidad de los materiales de acero inoxidable (1-3). Estos instrumentos son de dos a tres veces más flexibles que las limas de acero inoxidable (4). La aleación usada en la fabricación de instrumentos endodónticos consiste en aproximadamente 55\% níquel y 45\% titanio siendo llamada genéricamente 55-Nitinol (5). Desde entonces han revolucionado los convencionales diseños de los instrumentos, cambiando principalmente las secciones transversales y conicidades, en busca de instrumentos con mayor eficiencia de limpieza y conformación del sistema de conductos radiculares (6-9).

El NiTi proporciona aumento de la flexibilidad y resistencia a la fractura, características que mejoran el rendimiento de los instrumentos durante-la preparación biomecánica de conductos radiculares curvos (10-12). Las razones para la separación de los instrumentos rotatorios de NiTi incluyen variaciones de la anatomía del conducto, tales como fusión, curvaturas, dobles curvaturas, dilaceraciones o conductos divididos (13). Además, factores relacionados con el operador y la capacidad clínica son también importantes para la separación de instrumentos (14).

En 2006, Dentsply Maillefer (Ballaigues, Switzerland) lanzó al mercado el Sistema ProTaper Universal, uno de los más usados y estudiados. Este sistema es caracterizado por su diseño con conicidad variable a lo largo de su parte activa, convexidad triangular en su sección transversal, y puntas inactivas, disminuyendo con ello la fatiga del instrumento, las cargas de torsión y el potencial de fractura $(15,16)$.
El Sistema ProTaper Universal tiene un kit básico que contiene: un instrumento SX, especial para preparación cervical o desgaste anticurvatura; dos instrumentos para la conformación del conducto radicular, y tres instrumentos para la finalización de la preparación biomecánica: F1, F2 y F3. Esta secuencia sirve para la preparación de la mayoría de conductos radiculares (17).

No obstante, comercialmente existen instrumentos rotatorios de similares características, que aparentemente se asemejan al sistema ProTaper Universal, pero que son de dudosa procedencia, pudiendo comprometer la eficiencia de la instrumentación de los conductos radiculares. Por otro lado, no existen estudios que evalúen si estos instrumentos similares son de características macroscópicas, microscópicas y metalográficas iguales a los originales. Por lo tanto, es relevante hacer una evaluación comparativa con la finalidad de alertar a los endodoncistas clínicos sobre los riesgos de su uso.

Este estudio tiene como objetivo describir las características macroscópicas, microscópicas y de composición del sistema ProTaper Universal.

\section{MATERIAL Y MÉTODOS}

El análisis macroscópico se realizó a través de fotografía macro con cámara fotográfica profesional (EOS Rebel T5i; Canon Co.,Tokio Japón), objetivo macro (EF 100mm f/2.8L Macro; Canon, Tokio Japón), y flash circular (Macro Ring Lite; Canon Co., Tokio, Japan) en programación específica. La cámara fotográfica fue fijada perpendicularmente al fondo blanco utilizado, sobre el cual fueron posicionados individualmente los elementos descritos, previamente limpiados, para la obtención de las imágenes respectivas. 
Para el análisis microscópico, se utilizaron los instrumentos S1 y F1, los cuales fueron seccionados dos veces, a 5 y a $10 \mathrm{~mm}$ de la punta del instrumento con una máquina de corte (Isomet; Buehler, Lake Bluff, IL). El fragmento correspondiente a la punta del instrumento fue utilizado para el análisis microscópico de la parte activa, y el fragmento restante para el análisis microscópico de la sección transversal. Todos los fragmentos, fueron lavados con agua destilada, agitados en ultrasonido (Cristófoli, Campo Mourão, PR, Brasil) por 10 minutos, y posicionados individualmente en stubs metálicos y sin metalizar. Posteriormente se analizaron los fragmentos a través de Microscopía electrónica de barrido (EDX) (EVO 50, Zeiss, Cambridge, Reino Unido), operado en 20 $\mathrm{kV}$, comparando partes activas y secciones transversales de los instrumentos, con magnificación de 300 a 550X. Adicionalmente, se realizó la caracterización de los instrumentos a través de microanálisis por dispersión de energías de rayos-X (EDX) (6060; JEOL Ltda, Tokyo, Japan), operado en $20 \mathrm{kV}$, con los fragmentos de las partes activas utilizados en el análisis microscópico.

\section{RESULTADOS}

\section{Análisis macroscópico}

Inicialmente, se compararon las cajas de los sistemas ProTaper Universal y su homólogo adulterado (Figura 1), encontrándose diferencias en el diseño de las caras frontal y lateral de la caja. En la cara frontal de la caja adulterada, se puede observar la descripción del tipo de instrumento en varios idiomas en la parte superior (Figura $1 \mathrm{~B}$ ), que no se encuentra en la caja original (Figura $1 \mathrm{~A}$ ); el número de referencia de la parte lateral izquierda termina en 00 , a diferencia de la caja original que termina en 12; además la principal diferencia es la presencia del término "CE 0459" en la parte central (Figura $1 \mathrm{~B}$, flecha), como una especificación del producto, que tampoco es observada en la caja original. En la cara lateral de la caja adulterada, se puede observar una descripción resumida de los instrumentos (SX-F3) y no la descripción de cada uno en su respectiva posición (S1, S2, F1, F2, F3, SX) como en la caja original (Figura $1 \mathrm{~A}$ ); además se observa la longitud de los instrumentos (25mm), que no está presente en la caja original.

En la comparación macroscópica de los instrumentos SX (Figura 2), se encontraron diferencias principal- mente en el vástago del instrumento, como la variación del color dorado metálico y de las tonalidades del amarillo del tope (Figura A y B), asimismo, se distingue un acabado deficiente a nivel de la conexión al motor y la presencia de una diferencia de relieves (Figura 2B, flecha), que no está presente en el instrumento original. En la parte activa no se encuentran grandes diferencias macroscópicas (Figura 2 C, D y E), solo una ligera variación en la tonalidad del metal y la separación de la punta del instrumento (Figura 2 E), que ocurrió en su primer uso, revelando su poca resistencia, aparentemente por un diseño y composición que no correspondería al de un instrumento SX original.

La comparación macroscópica del resto de instrumentos fue similar, el vástago adulterado (Figura $3 \mathrm{~B})$ presenta una longitud menor, un color dorado metálico diferente, deficiencias en el acabado de la conexión del vástago al motor, diferente tonalidad en el color del tope y en el anillo de identificación. Además, existe diferencia en el formato, por un lado, el instrumento adulterado presenta un anillo de identificación cóncavo (Figura 3B, flecha), mientras que el anillo de identificación del instrumento original es totalmente recto. En la parte activa del instrumento adulterado (Figura $3 \mathrm{D}$ ), se puede observar una ligera diferencia en el color del metal en comparación con el instrumento original (Figura 3C), deficiencia en el acabado, una punta con diámetro mayor y ausencia de estrías, que caracterizan a los instrumentos de mayor calibre del sistema ProTaper Universal (Figura 3 C).

\section{Análisis microscópico}

La comparación microscópica mostró que los instrumentos S adulterados presentan ángulos de corte menos afilados (Figura 4 D) que los instrumentos originales, pero puntas más agudas (Figura 4E). La sección transversal de los instrumentos adulterados no presentan las convexidades triangulares características (Figura 4F, flecha) del sistema ProTaper Universal, quedando modificado el formato macroscópico propio del sistema. Los instrumentos $\mathrm{F}$ adulterados también presentan ángulos de corte menos afilados (Figura 5 D y E) que los originales. La sección transversal de los instrumentos adulterados presentan ángulos de corte romos y ausencia de convexidades triangulares características del sistema (Figura 5 C y F), modificando también el formato macroscópico (Figura $3 \mathrm{C}$ ). 

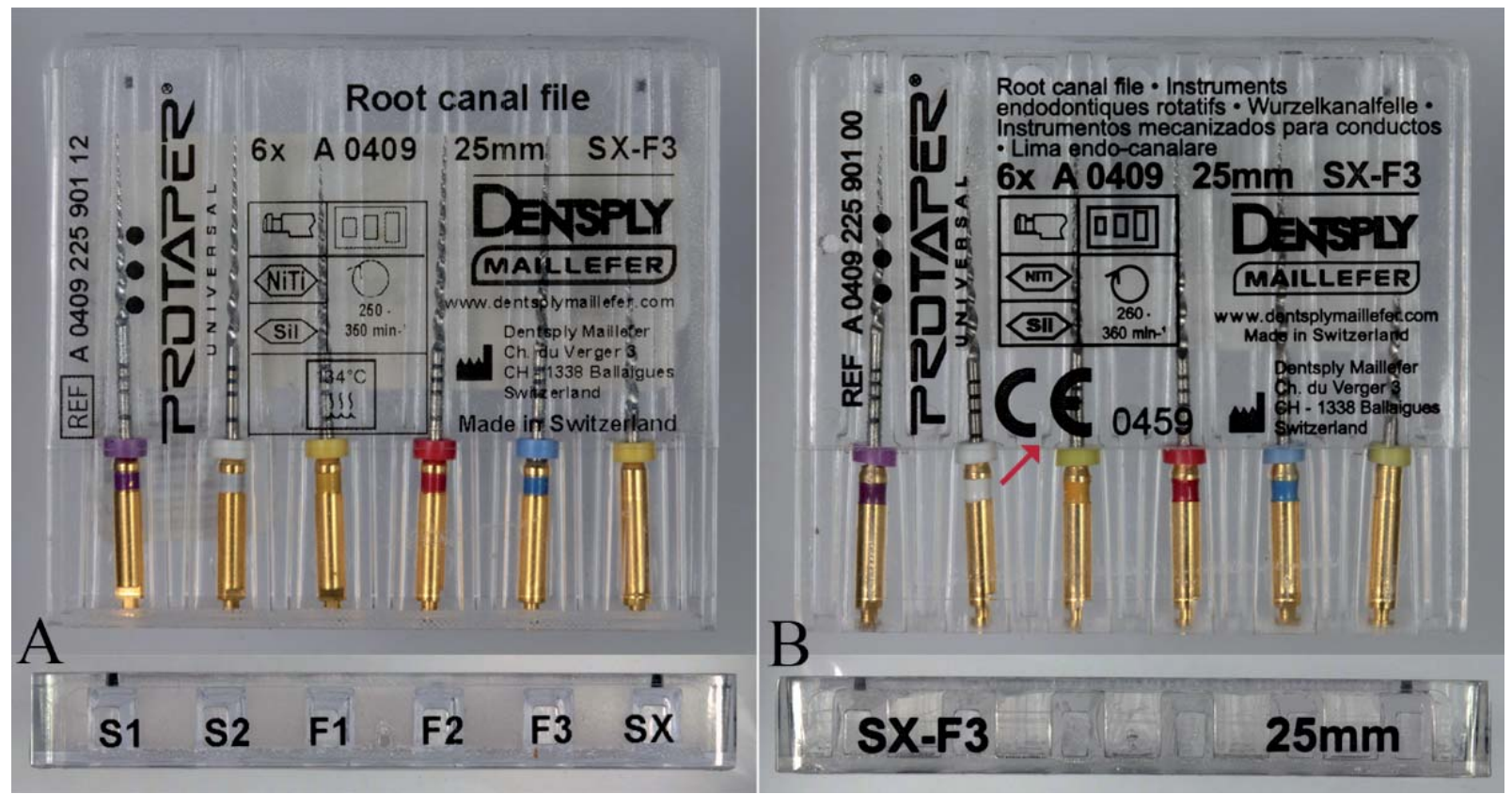

Figura 1. (A) Caja ProTaper Universal. (B) Caja ProTaper Universal adulterada; se pueden observar diferencias en el diseño (flecha).

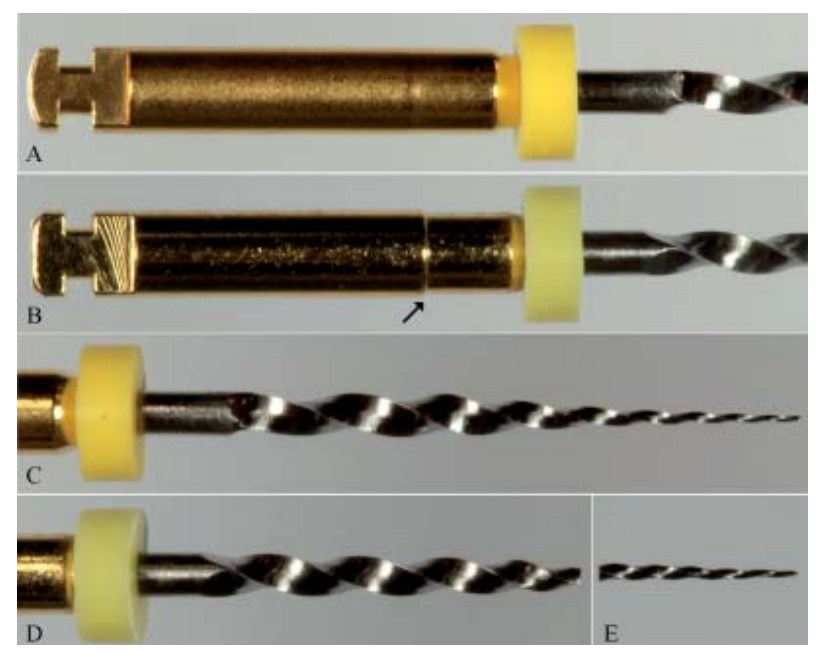

Figura 2. Análisis macroscópico de instrumento SX (A) Vástago original. (B) Vástago adulterado; se pueden observar diferencias en diseño, color y acabado (flecha). (C) Parte activa original. (D) Parte activa adulterada; se pueden observar diferencias en diseño, color y acabado. (E) Punta de instrumento separado.

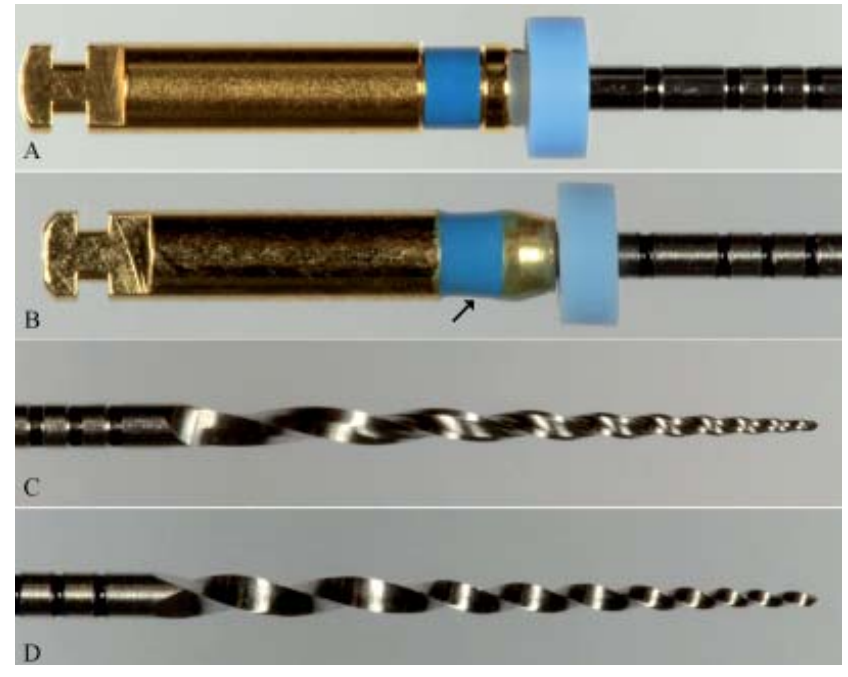

Figura 3. Análisis macroscópico de instrumento F3 (A) Vástago original. (B) Vástago adulterado; se pueden observar diferencias en diseño, color y acabado (flecha). (C) Parte activa original. (D) Parte activa adulterada; se pueden observar diferencias en diseño, color y acabado. 


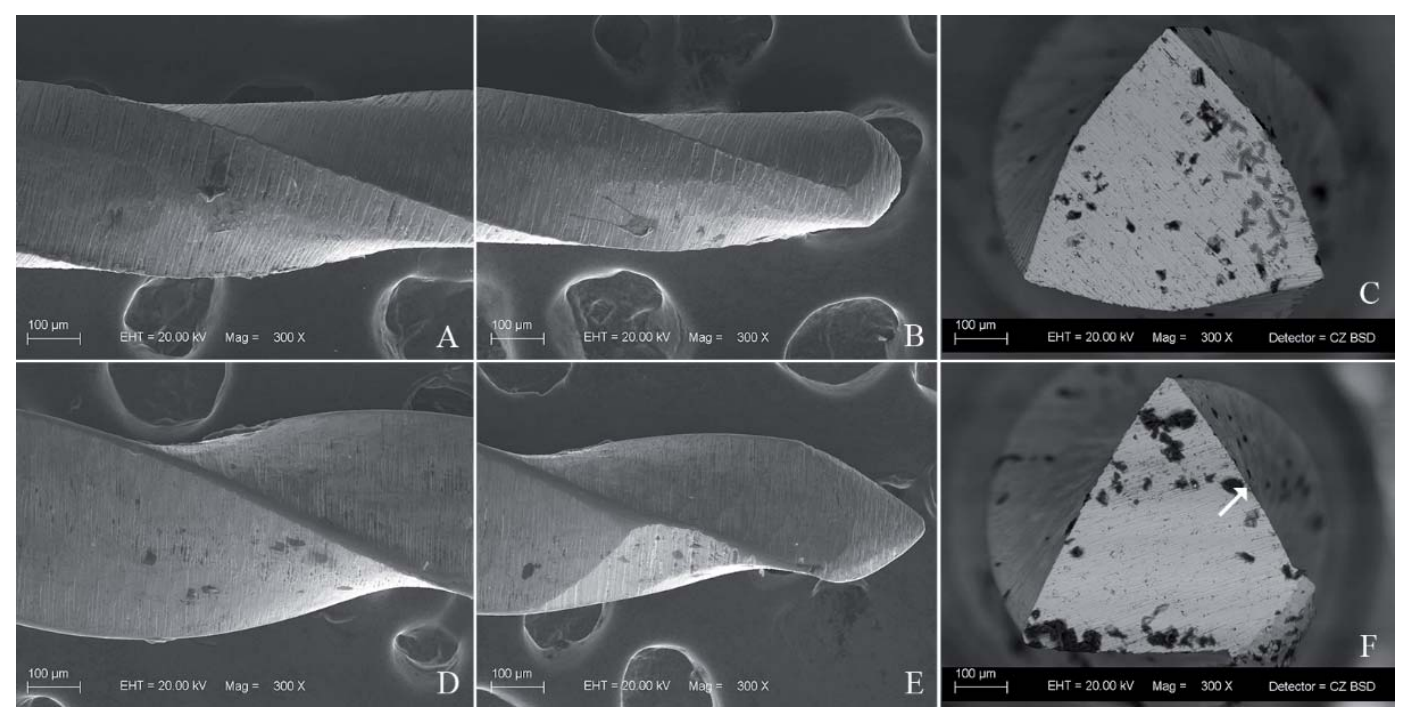

Figura 4. Análisis microscópico de instrumento S1. (A) y (B) Parte activa y punta de instrumento original. (C) Sección transversal de instrumento original. (D) y (E) Parte activa y punta de instrumento adulterado; se pueden observar diferencias en el diseño. (F) Sección transversal de instrumento adulterado, se pueden observar diferencias en el diseño (flecha).

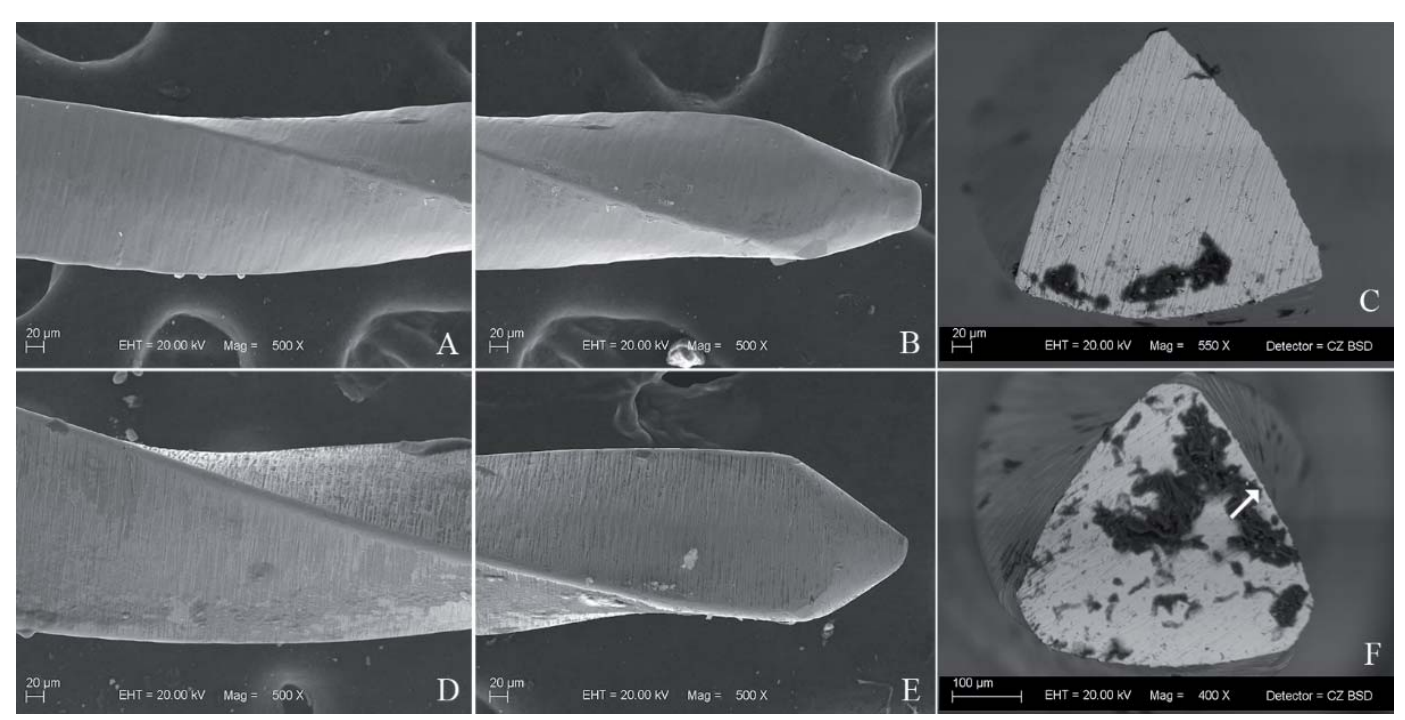

Figura 5. Análisis microscópico de instrumento F1. (A) y (B) Parte activa y punta de instrumento original. (C) Sección transversal de instrumento original. (D) y (E) Parte activa y punta de instrumento adulterada; se pueden observar diferencias en el diseño. (F) Sección transversal de instrumento adulterado, se pueden observar diferencias en el diseño (flecha). 


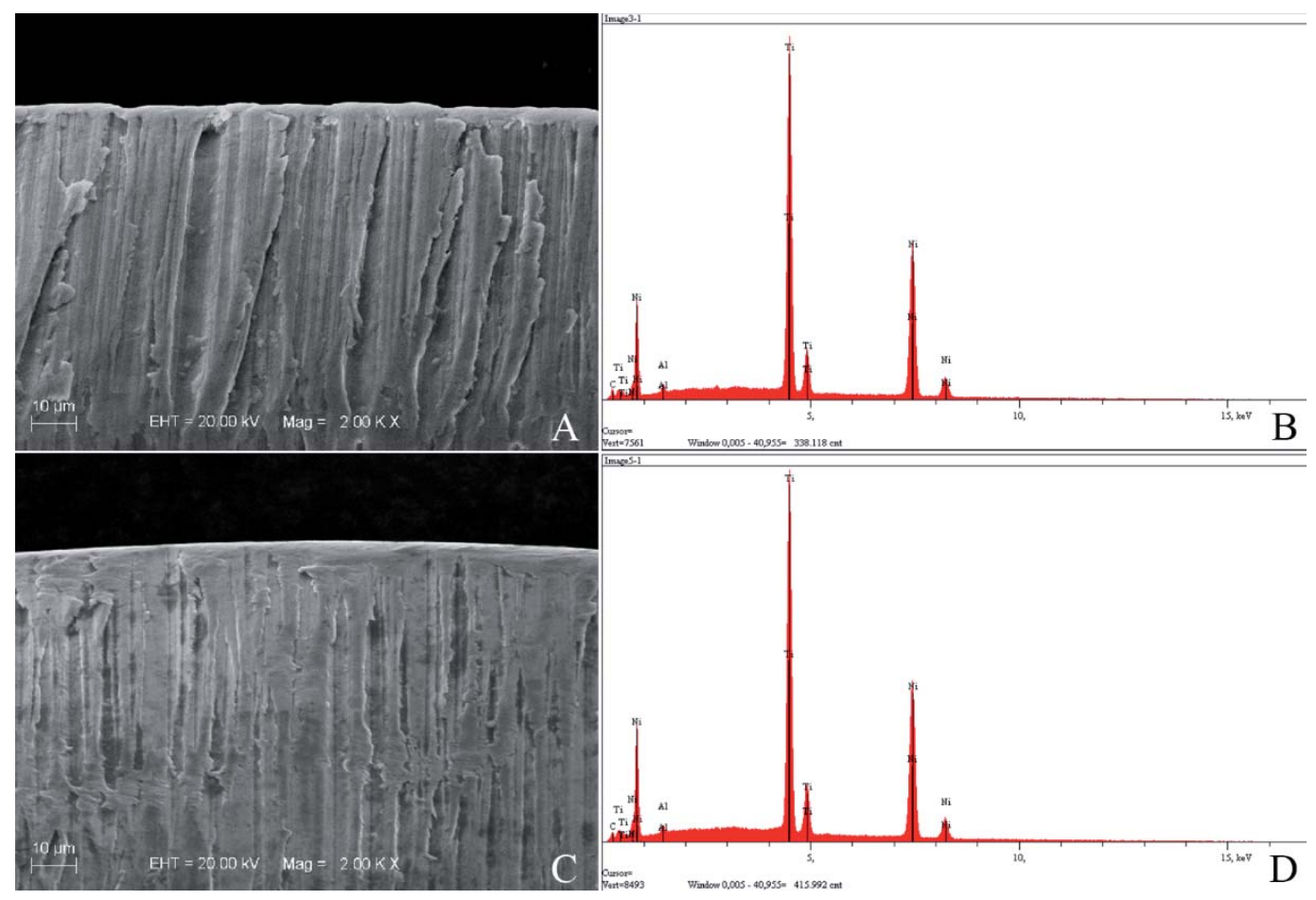

Figura 6. Análisis de composición de instrumento S1. (A) y (B) Instrumento ProTaper Universal original. (C) y (D) Instrumento adulterado. Ambas tienen una composición similar (Ni-Ti).

\section{Caracterización del material}

Al comparar la composición química de los instrumentos originales con sus homólogos adulterados, el análisis EDX mostró que los instrumentos originales con sus homólogos adulterados tienen una composición de Ni-Ti similar (Figura 6), descartando la hipótesis de una composición metálica diferente de los instrumento adulterados, planteada inicialmente por la separación prematura del instrumento SX adulterado (Figura $2 \mathrm{E}$ y $\mathrm{F}$ ) en su primer uso (el único que se usó clínicamente, antes de identificar que no eran las originales). Se puede deducir que los instrumentos adulterados fueron fabricados con una aleación de Ni-Ti similar al usado en el sistema ProTaper Universal original, pero que existen diferencias evidentes en el diseño y fabricación.

\section{DISCUSIÓN}

El tratamiento endodóntico está sujeto a diferentes accidentes y complicaciones como son la separación de los instrumentos dentro del conducto radicular, desvíos o perforaciones $(13,14)$; sin embargo, la preocupación reside en el aumento de la probabilidad de que estas complicaciones ocurran, no por la falta de habilidad y/o conocimientos del operador, ni por la complejidad anatómica del conducto, sino por una variable adicional inesperada, como podría ser la dudosa procedencia de instrumentos endodónticos. Estos instrumentos utilizan el nombre de marcas comerciales conocidas y ampliamente estudiadas, ya que su fabricación considera altos estándares de calidad y es respaldada por una serie de estudios internos del fabricante, así como de estudios independientes alrededor del mundo que avalan su viabilidad en la instrumentación de los conductos radiculares (12, 18-20)

En este estudio se realizaron tres metodologías diferentes para comparar los instrumentos ProTaper originales con los adulterados y fueron: análisis macroscópico con fotografía macro, análisis microscópico con microscopia electrónica de barrido y análisis de composición con microanálisis por dispersión de energías de rayos-X (EDX), siendo el análisis macroscópico en el que se pudieron identificar diferencias resaltantes que a simple vista podrían pasar por alto, sin embargo con el conocimiento de las características macroscópicas del sistema de limas con el 
que trabajamos, podríamos estar preparados para no ser sorprendidos.

Este trabajo sirve de base para la realización de estudios de análisis macroscópicos de los diversos sistemas de instrumento endodónticos disponibles en el mercado para conocimiento de las características singulares de cada uno.

\section{CONCLUSIONES}

Las mayores diferencias entre el sistema ProTaper Universal y su homólogo adulterado se presentaron en el análisis macroscópico. El análisis microscópico presentó diferencias principalmente en el formato de la sección transversal, y finalmente, la caracterización de materiales de los instrumentos demostró que son de composición similar.

\section{Correspondencia:}

Gabriela Castro Núñez

Correo electrónico: gabrielamcnunez@foar.unesp.br

\section{REFERENCIAS BIBLIOGRAFICAS}

1. Kim HC, Yum J, Hur B, Cheung GSP. Cyclic Fatigue and fracture characteristics of ground and twisted nickeltitanium rotary files. J Endod. 2010; 36(1):147-51.

2. Bonetti Filho I, Esberard RM, Leonardo RT, Del rio CE. Microscopic evaluation of three endodontic files prepostinstrumentation. J Endod. 1998; 24(7):461-4.

3. Alapati SB, Brantley WA, Svec TA, Powers JM, Mitchell JC. Scanning electron microscope observations of new and used nick el-titanium rotary files. J Endod. 2004; 29(10):667-9.

4. Vahid A, Roohi N, Zayeri F. A comparative study of four rotary NiTi instruments in preserving canal curvature, preparation time and change of working length. Aust Endod J. 2008; 32(5):1-5.

5. Vaudt J, Bitter K, Kielbassa AM. Rotary instruments in vitro. Endo. 2007; 1(3):189-293.

6. Foschi F, Nucci C, Montebugnoli L, et al. SEM evaluation of canal wall dentine following use of Mtwo and ProTaper NiTi rotary instruments. Int Endod J. 2004; 37:832-9.

7. Bergmans L, Van Cleynenbreugel J, Beullens M, Wevers M, Van Meerbeek B, Lambrechts P. Progressive versus constant tapered shaft design using NiTi rotary instruments. Int Endod J. 2003; 36(4):288-95.

8. Montoya JS, Trinidad JG, Loza JAM, Reygadas RC. Estudio comparativo del trabajo biomecánico del sistema Protaper y la instrumentación manual in vitro. Rev ADM. 2008; 65(3):126-32.
9. Vieira EP, França EC, Martins RC, Buono VTL, Bahia MGA. Influence of multiple clinical use on fatigue resistance of ProTaper rotary nickel-titanium instruments. Int Endod J. 2008; 41:163-72.

10. Sattapan B, Palamara JEA, Messer HH. Torque during canal instrumentation using rotary nickel-titanium files. J Endod. 2000 26(3):156-60.

11. Iqbal MK, Kohli MR, Kim JS. A retrospective clinical study of incidence of root canal instrument separation in an endodontics graduate program: a PennEndo database study. J Endod. 2006; 32(11):1048-52.

12. Lopes HP, Gambarra-Soares T, Elias CN, et al. Comparison of the mechanical properties of rotary instruments made of conventional nickel-titanium wire, M-ire, or nickel-titanium alloy in R-phase. J Endod. 2013; 39: 516-20.

13. Ruddle CJ. The ProTaper technique. Endod Topics. 2005;10:187-90.

14. Yared GM, Bou Dagher FE, Machtou P, Kulkarni GK. Influence of rotational speed, torque and operator proficiency on failure of Greater Taper files. Int Endod J. 2002;35(1):7-12.

15. West J. Progressive taper technology: rationale and clinical technique for the new ProTaper Universal System. Dent Today. 2006; 25:66-9.

16. Simon S, Lumley P, Tomson P, Pertot WJ, Machtou P. Protaper--hybrid technique. Dent Update. 2008; 35(2):110-2, 115-6.

17. Lopes HP, Siqueira JR JF. Endodontia: biologia e técnica. $4^{\circ}$ edição. Rio de Janeiro: Elsevier; 2015.p. 265-354.

18. Aguiar CM, Mendes DA, Câmara AC, Figueiredo JAP. Evaluation of the centreing ability of the ProTaper Universal $^{\mathrm{TM}}$ rotary system in curved roots in comparison to Nitiflex $^{\mathrm{TM}}$ files. Aust Endod J. 2009; 35: 174-9.

19. Kunert GG, Fontanella VRC, de Moura AAM, Barletta FB. Analysis of apical root transportation associated with ProTaper Universal F3 and F4 instruments by using digital subtraction radiography. J Endod. 2010; 36: 1052-5.

20. Aguiar CM, Mendes DA, Câmara AC, Figueiredo JAP. Assessment of canals walls after biomechanical preparation of root canals instrumented with ProTaper Universal $^{\text {TM }}$ rotary system. J Appl Oral Sci. 2009; 17: 590-5. Taper Universal adulterada; se pueden observar diferencias en el diseño (flecha).seño, color y acabado. 